\title{
Analytical Solutions of Boundary Values Problem of 2D and 3D Poisson and Biharmonic Equations by Homotopy Decomposition Method
}

\author{
Abdon Atangana ${ }^{1}$ and Adem Kilıçman ${ }^{2}$ \\ ${ }^{1}$ Institute for Groundwater Studies, Faculty of Natural and Agricultural Sciences, University of the Free State, \\ Bloemfontein 9300, South Africa \\ ${ }^{2}$ Department of Mathematics and Institute for Mathematical Research, University of Putra Malaysia, \\ 43400 Serdang, Selangor, Malaysia
}

Correspondence should be addressed to Adem Kılıçman; kilicman@yahoo.com

Received 13 June 2013; Accepted 18 August 2013

Academic Editor: Santanu Saha Ray

Copyright (C) 2013 A. Atangana and A. Kılıçman. This is an open access article distributed under the Creative Commons Attribution License, which permits unrestricted use, distribution, and reproduction in any medium, provided the original work is properly cited.

\begin{abstract}
The homotopy decomposition method, a relatively new analytical method, is used to solve the 2D and 3D Poisson equations and biharmonic equations. The method is chosen because it does not require the linearization or assumptions of weak nonlinearity, the solutions are generated in the form of general solution, and it is more realistic compared to the method of simplifying the physical problems. The method does not require any corrected function or any Lagrange multiplier and it avoids repeated terms in the series solutions compared to the existing decomposition method including the variational iteration method, the Adomian decomposition method, and Homotopy perturbation method. The approximated solutions obtained converge to the exact solution as $N$ tends to infinity.
\end{abstract}

\section{Introduction}

The numerical solution of Poisson equations and biharmonic equations is an important problem in numerical analysis. A vast arrangement of investigating effort has been published on the development of numerical solution of Poisson equations and biharmonic equations. The finite difference schemes of second and fourth order for the solution of Poisson's equation in polar coordinates have been derived by Mittal and Gahlaut [1]. A numerical method to interpolate the source terms of Poisson's equation by using B-spline approximation has been devised by Perrey-Debain and ter Morsche [2]. Sutmann and Steffen [3] proposed compact approximation schemes for the Laplace operator of fourth and sixth order; the schemes are based on a Padé approximation of the Taylor expansion for the discretized Laplace operator. Ge [4] used fourth-order compact difference discretization scheme with unequal mesh sizes in different coordinate directions to solve a 3D Poisson equation on a cubic domain. Gumerov and Duraiswami [5] developed a complete translation theory for the biharmonic equation in three dimensions. Khattar et al. [6] derived a fourth-order finite difference approximation based on arithmetic average discretization for the solution of three-dimensional nonlinear biharmonic partial differential equations on a 19-point compact stencil using coupled approach. Altas et al. [7] used multigrid and preconditioned Krylov iterative methods to solve three-dimensional nonlinear biharmonic partial differential equations. Jeon [8] derived scalar boundary integral equation formulas for both interior and exterior biharmonic equations with the Dirichlet boundary data. A spectral collocation method for numerically solving two-dimensional biharmonic boundaryvalue problems has been reported in [9]. An indirect radialbasis-function collocation method for numerically solving biharmonic boundary-value problems has been reported in [10]. A high-order boundary integral equation method for the solution of biharmonic equations has been presented in [11]. A Galerkin boundary node method for solving biharmonic problems was developed in [12]. An integral collocation approach based on Chebyshev polynomials for numerically 
solving biharmonic equations for the case of irregularly shaped domains has been developed by Mai-Duy et al. [13]. A numerical method, based on neural-network-based functions, for solving partial differential equations has been in [14]. Mai-Duy and Tanner [15] presented a collocation method based on a Cartesian grid and a 1D integrated radial basis function scheme for numerically solving partial differential equations in rectangular domains and Haar wavelet presented in [16]. The aim of this paper is to solve these problems via the homotopy decomposition method.

\section{Method}

In this study we follow the method of [17-20]. In order to illustrate the basic idea of this method we consider a general nonlinear nonhomogeneous partial differential equation with initial conditions of the following form

$$
\begin{array}{r}
\frac{\partial^{m} U(x, t)}{\partial t^{m}}=L(U(x, t))+N(U(x, t))+f(x, t), \\
m=1,2,3, \ldots,
\end{array}
$$

subject to the initial conditions

$$
\begin{array}{r}
\frac{\partial^{i} U(x, 0)}{\partial t^{i}}=f_{m}(x), \quad \frac{\partial^{m-1} U(x, 0)}{\partial t^{m-1}}=0, \\
i=0,1,2, \ldots, m-2,
\end{array}
$$

where $f$ is a known function, $N$ is the general nonlinear differential operator, and $L$ represents a linear differential operator. The method's first step here is to apply the inverse operator $\partial^{m} / \partial t^{m}$ of on both sides (1) to obtain

$$
\begin{aligned}
U(x, t)= & \sum_{k=0}^{m-1} \frac{t^{k}}{k !} \frac{d^{k} u(x, 0)}{d t^{k}} \\
& +\int_{0}^{t} \int_{0}^{t_{1}} \cdots \int_{0}^{t_{m-1}} L(U(x, \tau))+N(U(x, \tau)) \\
& +f(x, \tau) d \tau \cdots d t .
\end{aligned}
$$

The multi-integrals in (3) can be transformed to

$$
\begin{gathered}
\int_{0}^{t} \int_{0}^{t_{1}} \cdots \int_{0}^{t_{m-1}} L(U(x, \tau))+N(U(x, \tau)) \\
+f(x, \tau) d \tau \cdots d t_{1} \\
=\frac{1}{(m-1) !} \int_{0}^{t}(t-\tau)^{m-1} L(U(x, \tau))+N(U(x, \tau)) \\
\quad+f(x, \tau) d \tau .
\end{gathered}
$$

So that (3) can be reformulated as

$$
\begin{aligned}
& U(x, t) \\
& =\sum_{k=0}^{m-1} \frac{t^{k}}{k !}\left\{\frac{d^{k} u(x, 0)}{d t^{k}}\right\} \\
& +\frac{1}{(m-1) !} \int_{0}^{t}(t-\tau)^{m-1} L(U(x, \tau))+N(U(x, \tau)) \\
& +f(x, \tau) d \tau .
\end{aligned}
$$

Using the homotopy scheme the solution of the previous integral equation is given in a series form as

$$
\begin{gathered}
U(x, t, p)=\sum_{n=0}^{\infty} p^{n} U_{n}(x, t), \\
U(x, t)=\lim _{p \rightarrow 1} U(x, t, p)
\end{gathered}
$$

and the nonlinear term can be decomposed as

$$
N U(r, t)=\sum_{n=1}^{\infty} p^{n} \mathscr{H}_{n}(U),
$$

where $p \in(0,1]$ is an embedding parameter. $\mathscr{H}_{n}(U)$ is He's polynomials [21] that can be generated by

$$
\begin{array}{r}
\mathscr{H}_{n}\left(U_{0}, \ldots, U_{n}\right)=\frac{1}{n !} \frac{\partial^{n}}{\partial p^{n}}\left[N\left(\sum_{j=0}^{n} p^{j} U_{j}(x, t)\right)\right] \\
n=0,1,2 \ldots
\end{array}
$$

The homotopy decomposition method is obtained by the graceful coupling of decomposition method with He's polynomials and is given by

$$
\begin{aligned}
& \sum_{n=0}^{\infty} p^{n} U_{n}(x, t) \\
& =T(x, t) \\
& +p \frac{1}{(m-1) !} \int_{0}^{t}(t-\tau)^{m-1} \\
& \times\left[f(x, \tau)+L\left(\sum_{n=0}^{\infty} p^{n} U_{n}(x, \tau)\right)\right. \\
& \left.\quad+\sum_{n=0}^{\infty} p^{n} \mathscr{H}_{n}(U)\right] d \tau
\end{aligned}
$$

with

$$
T(x, t)=\sum_{k=0}^{m-1} \frac{t^{k}}{k !}\left\{\frac{d^{k} u(x, t)}{d t^{k}} \mid t=0\right\} .
$$

Comparing the terms of the same power of $p$ gives the solutions of various orders. The initial guess of the approximation is $T(x, t)$. Some further related results can be seen in [22-25].

Lemma 1 (see [17]). The complexity of the homotopy decomposition method is of order $O(n)$.

Proof. The number of computations including product, addition, subtraction, and division are as follows.

In step 2

$$
\begin{aligned}
& U_{0}: 0 \text { because it is obtained directly from the initial } \\
& \text { conditions } \\
& U_{1}: 3 \\
& \vdots \\
& U_{n}: 3 \text {. }
\end{aligned}
$$

Now in step 4 the total number of computations is equal to $\sum_{j=0}^{n} U_{j}(x, t)=3 n=O(n)$. 


\section{Solutions of the Main Problems}

Problem 1. Consider the following equation

$$
\begin{gathered}
\frac{\partial^{2} u}{\partial x^{2}}+\frac{\partial^{2} u}{\partial y^{2}}=\sin (\pi x) \sin (\pi y) ; \\
u(x, y)=0 \text { along the boundaries, } \quad 0 \leq x, y \leq 1 ;
\end{gathered}
$$

$$
u_{x}(0, y)=-\frac{\sin (y \pi)}{2 \pi} \text {. }
$$

The exact solution of the previous equation is given as

$$
u(x, y)=\frac{\sin (x \pi) \sin (\pi y)}{-2 \pi^{2}}
$$

In the view of the homotopy decomposition method, (11) can be first transformed to

$$
\begin{aligned}
u(x, y)= & u(0, y)-\frac{\sin (\pi y)}{2 \pi} x \\
& +\int_{0}^{x}(x-\tau)\left[\sin (\pi \tau) \sin (\pi y)-u_{y y}(\tau, y)\right] \\
& u(x, y, p)=\sum_{n=0}^{\infty} p^{n} u_{n}(x, y) .
\end{aligned}
$$

Following the decomposition techniques, we obtain the following equation

$$
\begin{aligned}
\sum_{n=0}^{\infty} p^{n} u_{n}(x, y) & \\
= & T(x, y) \\
& +p \int_{0}^{x}(x-\tau)[\sin (\pi \tau) \sin (\pi y) \\
& \left.\quad-\frac{\partial^{2}}{\partial y^{2}}\left[\sum_{n=0}^{\infty} p^{n} u_{n}(x, y)\right]\right] .
\end{aligned}
$$

Comparing the terms of the same power of $p$ leads to

$$
\begin{aligned}
& p^{0}: u_{0}(x, y)=-\frac{\sin (\pi y)}{2 \pi} x, \\
& p^{1}: u_{1}(x, y)=\int_{0}^{x}(x-\tau)\left[\sin (\pi \tau) \sin (\pi y)-\frac{\partial^{2}}{\partial y^{2}}\left[u_{0}\right]\right] d \tau, \\
& u_{1}(x, y)=0 \text { along the boundaries, } \\
& p^{2}: u_{2}(x, y)=\int_{0}^{x}(x-\tau)\left[-\frac{\partial^{2}}{\partial y^{2}}\left[u_{1}\right]\right] d \tau \text {, } \\
& p^{3}: u_{3}(x, y)=\int_{0}^{x}(x-\tau)\left[-\frac{\partial^{2}}{\partial y^{2}}\left[u_{2}\right]\right] d \tau \text {, }
\end{aligned}
$$

$$
\begin{aligned}
p^{n}: u_{n}(x, y) & =\int_{0}^{x}(x-\tau)\left[-\frac{\partial^{2} u_{n-1}}{\partial y^{2}}\right] d \tau \\
u_{n}(x, y) & =0 \text { along the boundaries. }
\end{aligned}
$$

The following solutions are obtained:

$$
\begin{gathered}
u_{0}(x, y)=-\frac{\sin (\pi y)}{2 \pi} x, \\
u_{1}(x, y)=\left[\frac{x}{\pi}-\frac{\pi x^{3}}{2 \times 3 !}\right] \sin (\pi y)-\frac{\sin (\pi \tau) \sin (\pi y)}{\pi^{2}},
\end{gathered}
$$$$
u_{2}(x, y)=\left[-\frac{x}{\pi}+\frac{\pi x^{3}}{6}-\frac{\pi^{3} x^{5}}{240}\right] \sin (\pi y)+\frac{\sin (\pi \tau) \sin (\pi y)}{\pi^{2}},
$$$$
u_{3}(x, y)=\left[\frac{x}{\pi}-\frac{\pi x^{3}}{6}+\frac{\pi^{3} x^{5}}{120}-\frac{\pi^{5} x^{7}}{10080}\right] \sin (\pi y)
$$$$
-\frac{\sin (\pi \tau) \sin (\pi y)}{\pi^{2}}
$$

$u_{4}(x, y)$

$$
\begin{aligned}
= & {\left[-\frac{x}{\pi}+\frac{\pi x^{3}}{6}-\frac{\pi^{3} x^{5}}{120}+\frac{\pi^{5} x^{7}}{5040}-\frac{\pi^{7} x^{9}}{725760}\right] \sin (\pi y) } \\
& +\frac{\sin (\pi \tau) \sin (\pi y)}{\pi^{2}},
\end{aligned}
$$

$$
\begin{aligned}
& u_{5}(x, y) \\
& =\left[\frac{x}{\pi}-\frac{\pi x^{3}}{6}+\frac{\pi^{3} x^{5}}{120}-\frac{\pi^{5} x^{7}}{5040}+\frac{\pi^{7} x^{9}}{362880}-\frac{\pi^{9} x^{11}}{79833600}\right] \sin (\pi y) \\
& -\frac{\sin (\pi \tau) \sin (\pi y)}{\pi^{2}}, \\
& u_{6}(x, y) \\
& =\left[-\frac{x}{\pi}+\frac{\pi x^{3}}{6}-\frac{\pi^{3} x^{5}}{120}+\frac{\pi^{5} x^{7}}{5040}-\frac{\pi^{7} x^{9}}{362880}\right. \\
& \left.+\frac{\pi^{9} x^{11}}{39916800}-\frac{\pi^{11} x^{13}}{12454041600}\right] \sin (\pi y) \\
& +\frac{\sin (\pi \tau) \sin (\pi y)}{\pi^{2}}, \\
& u_{7}(x, y) \\
& =\left[\frac{x}{\pi}-\frac{\pi x^{3}}{6}+\frac{\pi^{3} x^{5}}{120}-\frac{\pi^{5} x^{7}}{5040}+\frac{\pi^{7} x^{9}}{362880}-\frac{\pi^{9} x^{11}}{39916800}\right. \\
& \left.+\frac{\pi^{11} x^{13}}{6227020800}-\frac{\pi^{13} x^{15}}{2615348736000}\right] \sin (\pi y) \\
& -\frac{\sin (\pi \tau) \sin (\pi y)}{\pi^{2}} .
\end{aligned}
$$


TABLE 1: Evaluation of numerical errors for $N=4$.

\begin{tabular}{ccccc}
\hline$x$ & $Y$ & $u(x, y)$ exact & $u(x, y) N=4$ & Error \\
\hline \multirow{4}{*}{0.25} & 0.25 & -0.0253303 & -0.0253303 & $6.27007 \cdot 10^{-11}$ \\
& 0.5 & -0.0358224 & -0.0358224 & $8.86722 \cdot 10^{-11}$ \\
& 0.75 & -0.0253303 & -0.0253303 & $6.27007 \cdot 10^{-11}$ \\
& 0.95 & -0.00560387 & -0.00560387 & $1.38714 \cdot 10^{-11}$ \\
\hline \multirow{4}{*}{0.5} & 0.25 & -0.0358224 & -0.0358224 & $1.26904 \cdot 10^{-11}$ \\
& 0.5 & -0.0506606 & -0.0506604 & $1.79469 \cdot 10^{-7}$ \\
& 0.75 & -0.0358224 & -0.0358223 & $1.26904 \cdot 10^{-11}$ \\
& 0.95 & -0.00792506 & -0.00792506 & $2.80752 \cdot 10^{-8}$ \\
\hline \multirow{4}{*}{0.75} & 0.25 & -0.0253303 & -0.0253195 & $1.07646 \cdot 10^{-5}$ \\
& 0.5 & -0.0358224 & -0.0358072 & $1.52235 \cdot 10^{-5}$ \\
& 0.75 & -0.0253303 & -0.0253195 & $1.07646 \cdot 10^{-5}$ \\
& 0.95 & -0.00560387 & -0.00560148 & $2.38148 \cdot 10^{-6}$ \\
\hline \multirow{6}{*}{0.95} & 0.25 & -0.00560387 & -0.00546191 & 0.000141956 \\
& 0.5 & -0.00792506 & -0.00772431 & 000200756 \\
& 0.75 & -0.00560387 & -0.00546191 & 0.000141956 \\
& 0.95 & -0.00123975 & -0.00120835 & $3.14051 \cdot 10^{-5}$ \\
\hline
\end{tabular}

In the same manner one can obtain the rest of the components. But for eight terms were computed and the asymptotic solution is given by

$$
\begin{aligned}
u_{N=8}(x, y) & \\
= & {\left[\frac{x}{2 \pi}-\frac{\pi x^{3}}{2 \times 3 !}+\frac{\pi^{3} x^{5}}{2 \times 5 !}-\frac{\pi^{5} x^{7}}{2 \times 7 !}+\frac{\pi^{7} x^{9}}{2 \times 9 !}\right.} \\
& \left.-\frac{\pi^{9} x^{11}}{2 \times 11 !}+\frac{\pi^{11} x^{13}}{2 \times 13 !}-\frac{\pi^{13} x^{15}}{2 \times 15 !}\right] \sin (\pi y) \\
& -\frac{1}{\pi^{2}} \sin (x \pi) \sin (y \pi) .
\end{aligned}
$$

Therefore in general for any $N>8$ we have

$$
\begin{gathered}
u_{N=n}(x, y)=\left[\frac{1}{2 \pi^{2}} \sum_{n=0}^{N} \frac{(-1)^{n}(x \pi)^{2 n+1}}{(2 n+1) !}\right] \sin (\pi y) \\
-\frac{1}{\pi^{2}} \sin (x \pi) \sin (y \pi), \\
\lim _{N \rightarrow \infty} u_{N}(x, y)=\frac{1}{2 \pi^{2}} \sin (\pi x) \sin (\pi y) \\
-\frac{1}{\pi^{2}} \sin (x \pi) \sin (y \pi) \\
=-\frac{1}{2 \pi^{2}} \sin (x \pi) \sin (y \pi) .
\end{gathered}
$$

This is the exact solution of the problem. Figures 1 and 2 show the comparison of the exact solution and the approximated one for $N=4$. The approximate solution and the exact solution are compared in Figures 1 and 2, respectively.

The numerical errors for $N=4$ are evaluated in Table 1 .

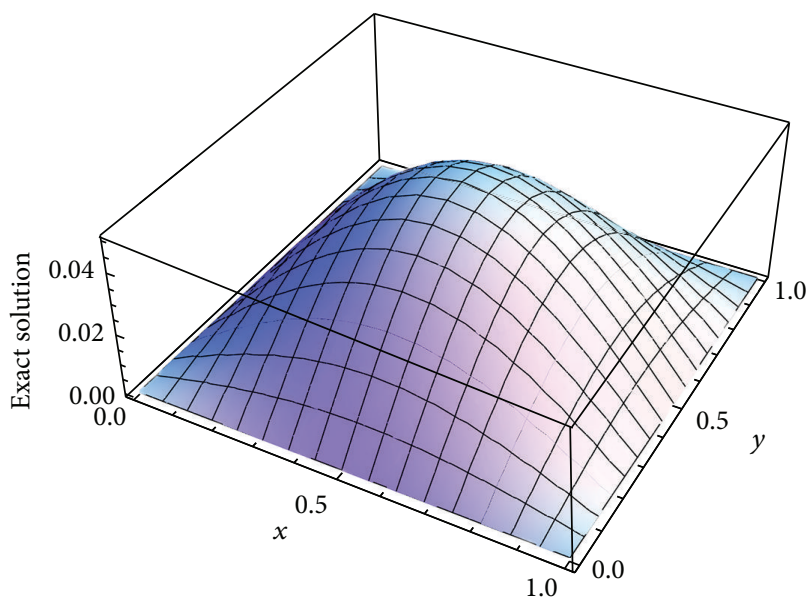

FIGURE 1: Exact solution.

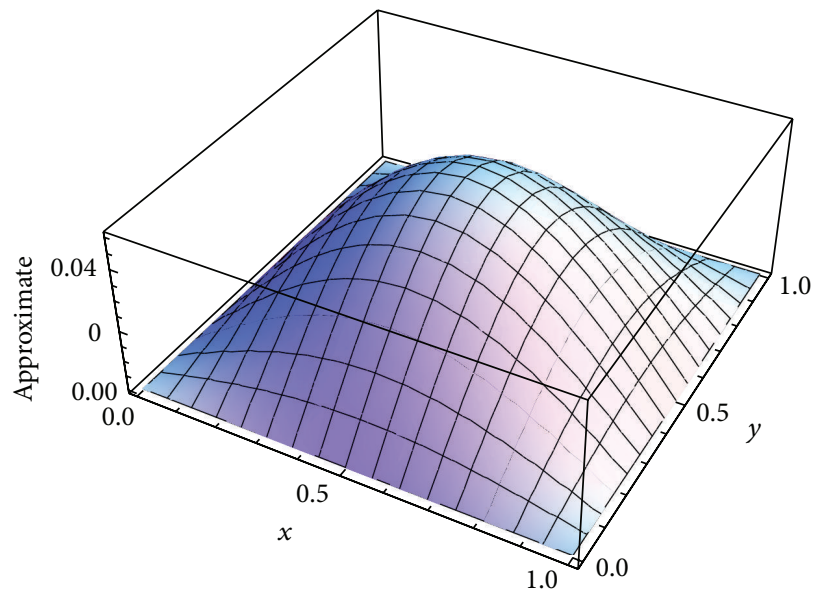

FIgURE 2: Approximated solution for the 4 first terms.

Problem 2. Consider 3D Poisson equation:

$$
\begin{gathered}
\frac{\partial^{2} u}{\partial x^{2}}+\frac{\partial^{2} u}{\partial y^{2}}+\frac{\partial^{2} u}{\partial z^{2}}=\sin (\pi x) \sin (\pi y) \sin (\pi z), \\
u(x, y, z)=0 \text { along the boundaries, } \quad 0 \leq x, y \leq 1 .
\end{gathered}
$$

Following the discussion presented earlier we obtain the following set of integral equations:

$$
\begin{aligned}
p^{0}: u_{0}(x, y) & =-\frac{\sin (\pi y) \sin (\pi z)}{3 \pi} x, \\
p^{1}: u_{1}(x, y) & \int_{0}^{x}(x-\tau)\left[\sin (\pi \tau) \sin (\pi y) \sin (\pi z)-\frac{\partial^{2} u_{0}}{\partial y^{2}}\right] d \tau, \\
& =\int_{0}^{x}(x-\tau)\left[-\frac{\partial^{2} u_{n-1}}{\partial y^{2}}\right] d \tau, \\
p^{n}: u_{n}(x, y) & \\
u_{n}(x, y) & =0 \text { along the boundaries, } \quad n \geq 2 .
\end{aligned}
$$


The following solutions are obtained:

$$
\begin{aligned}
& u_{0}(x, y, z)=-\frac{\sin (\pi y) \sin (\pi z)}{3 \pi} x, \\
& u_{1}(x, y, z)=\left[\frac{1}{\pi} x-\frac{\pi x^{3}}{9}\right] \sin (\pi y) \sin (\pi z) \\
& -\frac{\sin (\pi \tau) \sin (\pi y) \sin (\pi z)}{\pi^{2}}, \\
& u_{2}(x, y, z)=\left[-\frac{2}{\pi} x+\frac{\pi x^{3}}{9}-\frac{\pi^{3} x^{5}}{90}\right] \sin (\pi y) \sin (\pi z) \\
& +\frac{2 \sin (\pi \tau) \sin (\pi y) \sin (\pi z)}{\pi^{2}} \\
& u_{3}(x, y, z) \\
& =\left[\frac{4}{\pi} x-\frac{2 \pi x^{3}}{3}+\frac{\pi^{3} x^{5}}{30}-\frac{\pi^{5} x^{7}}{1890}\right] \sin (\pi y) \sin (\pi z) \\
& -\frac{4 \sin (\pi \tau) \sin (\pi y) \sin (\pi z)}{\pi^{2}}, \\
& u_{4}(x, y, z) \\
& =\left[-\frac{8}{\pi} x+\frac{4 \pi x^{3}}{3}-\frac{\pi^{3} x^{5}}{15}+\frac{\pi^{5} x^{7}}{630}\right] \sin (\pi y) \sin (\pi z) \\
& +\frac{8 \sin (\pi \tau) \sin (\pi y) \sin (\pi z)}{\pi^{2}}, \\
& u_{5}(x, y, z)=\left[\frac{16}{\pi} x-\frac{8 \pi x^{3}}{3}+\frac{2 \pi^{3} x^{5}}{15}-\frac{\pi^{5} x^{7}}{315}\right. \\
& \left.+\frac{\pi^{7} x^{9}}{22680}-\frac{\pi^{7} x^{11}}{3742200}\right] \sin (\pi y) \sin (\pi z) \\
& -\frac{16 \sin (\pi \tau) \sin (\pi y) \sin (\pi z)}{\pi^{2}} .
\end{aligned}
$$

In the same manner one can obtain the rest of the components. But for six terms were computed and the asymptotic solution is given by

$$
\begin{aligned}
u(x, y, z)_{N=6}= & {\left[\frac{x}{3 \pi}-\frac{\pi x^{3}}{18}+\frac{\pi^{3} x^{5}}{360}-\frac{\pi^{5} x^{7}}{15120}+\frac{\pi^{7} x^{9}}{1088640}\right.} \\
& \left.-\frac{\pi^{9} x^{11}}{119750400}\right] \sin (\pi y) \sin (\pi z) \\
& -\frac{2 \sin (\pi \tau) \sin (\pi y) \sin (\pi z)}{\pi^{2}},
\end{aligned}
$$

$$
\begin{aligned}
& u(x, y, z)_{N=6}=\frac{1}{3 \pi^{2}}\left[\frac{x}{3 \pi}-\frac{\pi x^{3}}{18}+\frac{\pi^{3} x^{5}}{360}-\frac{\pi^{5} x^{7}}{15120}+\frac{\pi^{7} x^{9}}{1088640}\right. \\
& \left.-\frac{\pi^{9} x^{11}}{119750400}\right] \sin (\pi y) \sin (\pi z) \\
& -\frac{2 \sin (\pi \tau) \sin (\pi y) \sin (\pi z)}{3 \pi^{2}} \\
& u(x, y, z)_{N=6}=\frac{1}{3 \pi^{2}}\left[\pi x-\frac{(\pi x)^{3}}{3 !}+\frac{(\pi x)^{5}}{5 !}-\frac{(\pi x)^{7}}{7 !}\right. \\
& \left.+\frac{(\pi x)^{9}}{9 !}-\frac{(\pi x)^{3}}{11 !}\right] \sin (\pi y) \sin (\pi z) \\
& -\frac{2 \sin (\pi \tau) \sin (\pi y) \sin (\pi z)}{3 \pi^{2}} \text {. }
\end{aligned}
$$

Therefore, for any $n \geq 6$, the partial sum is given as

$$
\begin{aligned}
u_{N=n}(x, y, z)= & \frac{1}{3 \pi^{2}}\left[\sum_{k=1}^{N} \frac{(-1)^{k}(\pi x)^{2 k+1}}{(2 k+1) !}\right] \sin (\pi y) \sin (\pi z) \\
& -\frac{2 \sin (\pi \tau) \sin (\pi y) \sin (\pi z)}{3 \pi^{2}} .
\end{aligned}
$$

Thus

$$
\begin{aligned}
u(x, y, z)= & \lim _{N \rightarrow \infty} u_{N=n}(x, y, z) \\
= & \frac{\sin (\pi \tau) \sin (\pi y) \sin (\pi z)}{3 \pi^{2}} \\
& -\frac{2 \sin (\pi \tau) \sin (\pi y) \sin (\pi z)}{3 \pi^{2}} \\
= & -\frac{\sin (\pi \tau) \sin (\pi y) \sin (\pi z)}{3 \pi^{2}} .
\end{aligned}
$$

And this is the exact solution to the problem. One can evaluate error committed by choosing the $N$ first terms in the series solutions, in the same manner as in Table 1. The accuracy of the results is estimated by error function

$$
R_{N}(x, y, z)=\left|u_{N}(x, y, z)-u(x, y, z)\right| .
$$

Problem 3. Let us consider the following biharmonic equation

$$
\frac{d^{4} u(x)}{d x^{4}}+4 u(x)=0
$$

for which the exact solution is

$$
u(x)=\frac{\operatorname{Exp}[1-x] \cos [x]}{\cos [1]}
$$

The aim of this part is to compare the numerical results obtained via HDM and the method used in [26]. 
TABLE 2: Comparison of the HDM and [1] results with the exaction solution for $N=6$.

\begin{tabular}{lccccc}
\hline$x$ & HDM & Exact & ADM & Err for HDM & Err for ADM \\
\hline-1.0 & 7.38906 & 7.38906 & 7.38906 & $6.78 E-16$ & $8.88 E-16$ \\
-0.6 & 7.56598 & 7.56598 & 7.56598 & $4.76 E-12$ & $7.96 E-12$ \\
-0.2 & 6.02244 & 6.02244 & 6.02244 & $0.015 E-11$ & $1.46 E-11$ \\
0.2 & 4.03696 & 4.03696 & 4.03696 & $0.017 E-11$ & $1.80 E-11$ \\
0.6 & 2.27883 & 2.27883 & 2.27883 & $0.015 E-11$ & $1.46 E-11$ \\
1.0 & 1.0 & 1.0 & 1.0 & $1.24 E-15$ & $2.22 E-15$ \\
\hline
\end{tabular}

Applying the steps involved in the HDM, we arrive at the following:

$$
\begin{aligned}
& u_{0}(x)=e \operatorname{Sec}(1)\left(1-x+\frac{x^{3}}{3}\right) \\
& u_{1}(x)=-e \operatorname{Sec}(1)\left[\frac{x^{4}}{4}-\frac{x^{5}}{20}+\frac{x^{7}}{420}\right] \\
& u_{2}(x)=-e \operatorname{Sec}(1)\left[-\frac{x^{8}}{1120}+\frac{x^{9}}{10080}-\frac{x^{11}}{554400}\right] \text {, } \\
& u_{3}(x)=-e \operatorname{Sec}(1)\left[\frac{x^{12}}{2217600}-\frac{x^{13}}{64864800}+\frac{x^{15}}{1135134000}\right] \text {, } \\
& u_{4}(x) \\
& =-e \operatorname{Sec}(1)\left[-\frac{x^{16}}{16144128000}+\frac{x^{17}}{274450176000}\right. \\
& \left.-\frac{x^{19}}{46930980096000}\right] \\
& u_{5}(x) \\
& =-e \operatorname{Sec}(1)\left[\frac{x^{20}}{312873200640000}-\frac{x^{21}}{6570337213440000}\right. \\
& \left.+\frac{x^{23}}{1662295315000320000}\right] \text {. }
\end{aligned}
$$

In the same manner, one can obtain the remaining term by using the following recursive formula:

$$
u_{n+1}(x)=-\int_{0}^{x}(x-t)^{3} u_{n}(t) d t
$$

In this paper we consider only the first six terms of the series solution as follows:

$$
u_{N=6}=\sum_{n=0}^{5} u_{n}(x)
$$

To access the accuracy of the method used in paper, we compare in Table 2 the numerical results of the above equation, the solution obtained in [26] with the exact solution.
Problem 4. We consider the $2 \mathrm{D}$ biharmonic equation

$$
\frac{\partial^{4} u}{\partial x^{4}}+2 \frac{\partial^{4} u}{\partial x^{2} \partial y^{2}}+\frac{\partial^{4} u}{\partial x^{4}}=\sin (3 \pi x) \sin (3 \pi y), \quad 0 \leq x, y \leq 1,
$$

subject to the initial conditions:

$$
\begin{gathered}
\frac{\partial u(x, y)}{\partial x} \mid(x=0)=\frac{\sin (3 \pi y)}{108 \pi^{3}}, \quad \partial_{x, x} u(0, y)=0 \\
\partial_{x, x, x} u(0, y)=-\frac{\sin (3 \pi y)}{12 \pi} .
\end{gathered}
$$

In the view of the homotopy decomposition method, the following integral equations are obtained:

$$
\begin{aligned}
& p^{0}: u_{0}(x, y)=\frac{\sin (3 \pi y)}{108 \pi^{3}} x-\frac{\sin (3 \pi y)}{12 \pi} \frac{x^{3}}{3 !}, \\
& p^{1}: u_{1}(x, y) \\
& =\int_{0}^{x}(x-\tau)\left[\sin (\pi \tau) \sin (\pi y)-\frac{\partial^{2} u_{0}}{\partial y^{2}}-2 \frac{\partial^{4} u_{0}}{\partial x^{2} \partial y^{2}}\right] d \tau, \\
& p^{n}: u_{n}(x, y)=\int_{0}^{x}(x-\tau)\left[-\frac{\partial^{2} u_{n-1}}{\partial y^{2}}-2 \frac{\partial^{4} u_{0}}{\partial x^{2} \partial y^{2}}\right] d \tau, \\
& u_{n}(x, y)=0 \text { along the boundaries, } \quad n \geq 2 .
\end{aligned}
$$

It is worth noting that if the zeroth component $u_{0}(x, y)$ is defined, then the remaining components $n \geq 1$ can be completely determined such that each term is determined by using the previous terms, and the series solutions are thus entirely determined. Finally, the solution $u(x, y)$ is approximated for $n=4$ :

$$
\begin{aligned}
& u(x, y) \\
& =\sin (3 \pi y)\left[\frac{x}{108 \pi^{3}}-\frac{x^{3}}{72 \pi}+\frac{\pi x^{5}}{160}-\frac{3 \pi^{3} x^{7}}{2240}+\frac{3 \pi^{5} x^{9}}{17920}\right. \\
& \left.\quad-\frac{27 \pi^{7} x^{11}}{1971200}+\frac{81 \pi^{9} x^{13}}{102502400}-\frac{81 \pi^{11} x^{15}}{7175168000}\right] \\
& \begin{array}{c}
u(x, y) \\
=\frac{\sin (3 \pi y)}{324 \pi^{4}}\left[3 \pi x-\frac{(3 \pi x)^{3}}{3 !}+\frac{(3 \pi x)^{5}}{5 !}-\frac{(3 \pi x)^{7}}{7 !}+\frac{(3 \pi x)^{9}}{9 !}\right. \\
\left.-\frac{(3 \pi x)^{11}}{11 !}+\frac{(3 \pi x)^{13}}{13 !}-\frac{(3 \pi x)^{15}}{15 !}\right] .
\end{array}
\end{aligned}
$$

Therefore for any $N \geq 4$ we have the following:

$$
u_{N}(x, y)=\frac{\sin (3 \pi y)}{324 \pi^{4}} \sum_{n=0}^{N} \frac{(3 \pi x)^{2 n+1}}{(2 n+1) !}
$$




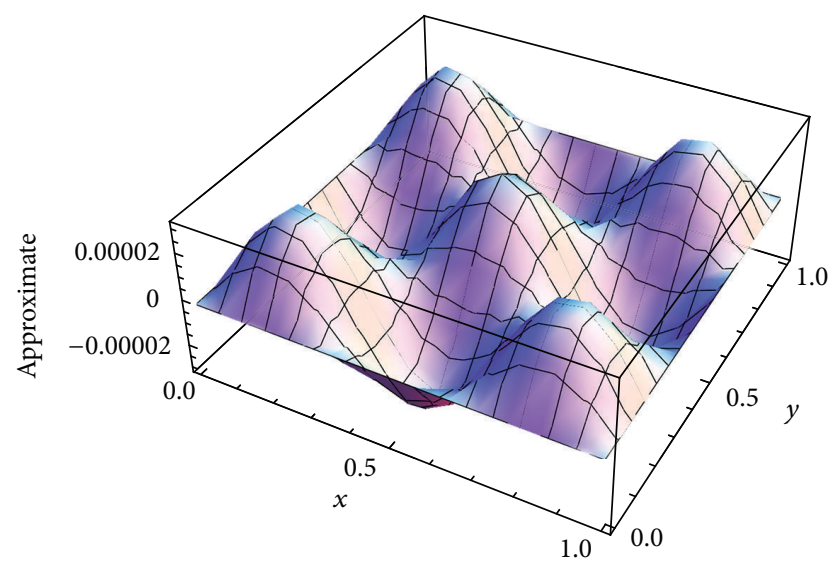

FIGURE 3: Analytical solution.

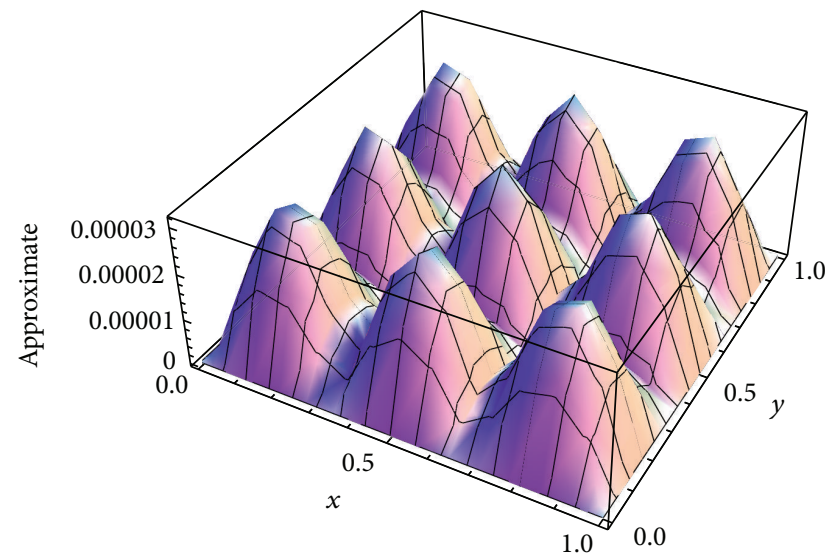

FIGURE 4: Absolute value of the solution.

Thus

$$
\lim _{N \rightarrow \infty} u_{N}(x, y)=\frac{\sin (3 \pi y) \sin (3 \pi x)}{324 \pi^{4}} .
$$

The exact solution of (31) is given by

$$
\frac{\sin (3 \pi y) \sin (3 \pi x)}{324 \pi^{4}}=u(x, y) .
$$

Figures 3 and 4 are the graphical representation of the previous solution. We have plotted the solution for (31) in Figure 3 and showed absolute value of the solution in Figure 4.

Theorem 2. Let $m$ be a nonzero natural number and let $(x, y) \in[0,1] \times[0,1]$; then two dimensional biharmonic equation of form

$$
\frac{\partial^{4} u}{\partial x^{4}}+2 \frac{\partial^{4} u}{\partial x^{2} \partial y^{2}}+\frac{\partial^{4} u}{\partial y^{4}}=\sin (m \pi x) \sin (m \pi y)
$$

with $u(x, y)=0$ along the boundaries has an exact solution as follows

$$
u(x, y)=\frac{\sin (m \pi x) \sin (m \pi y)}{4 m^{4} \pi^{4}}
$$

Proof. Use the step of the homotopy decomposition method.

Problem 5. We consider the 3D biharmonic equation:

$$
\begin{gathered}
\frac{\partial^{4} u}{\partial x^{4}}+2 \frac{\partial^{4} u}{\partial x^{2} \partial y^{2}}+2 \frac{\partial^{4} u}{\partial x^{2} \partial z^{2}}+2 \frac{\partial^{4} u}{\partial z^{2} \partial y^{2}}+\frac{\partial^{4} u}{\partial y^{4}}+\frac{\partial^{4} u}{\partial z^{4}} \\
=\sin (\pi x) \sin (\pi y) \sin (\pi z), \\
0 \leq x, y, z \leq 1, \\
u=0, \quad u_{x, x}=u_{y, y}=u_{z, z}=0 .
\end{gathered}
$$

In the view of the homotopy decomposition method, the following integral equations are obtained:

$$
\begin{aligned}
& p^{0}: u_{0}(x, y)=\frac{\sin (3 \pi y)}{108 \pi^{3}} x-\frac{\sin (3 \pi y)}{12 \pi} \frac{x^{3}}{3 !}, \\
& p^{1}: u_{1}(x, y) \\
& =\int_{0}^{x}(x-\tau)[\sin (\pi \tau) \sin (\pi y) \sin (\pi z) \\
& -2 \frac{\partial^{4} u_{0}}{\partial x^{2} \partial y^{2}}-2 \frac{\partial^{4} u_{0}}{\partial x^{2} \partial z^{2}} \\
& \left.-2 \frac{\partial^{4} u_{0}}{\partial z^{2} \partial y^{2}}-\frac{\partial^{4} u_{0}}{\partial y^{4}}-\frac{\partial^{4} u_{0}}{\partial z^{4}}\right] d \tau, \\
& p^{n}: u_{n}(x, y) \\
& =\int_{0}^{x}(x-\tau)\left[-2 \frac{\partial^{4} u_{n-1}}{\partial x^{2} \partial y^{2}}-2 \frac{\partial^{4} u_{n-1}}{\partial x^{2} \partial z^{2}}\right. \\
& \left.-2 \frac{\partial^{4} u_{n-1}}{\partial z^{2} \partial y^{2}}-\frac{\partial^{4} u_{n-1}}{\partial y^{4}}-\frac{\partial^{4} u_{n-1}}{\partial z^{4}}\right] d \tau, \\
& u_{n}(x, y)=0 \text { along the boundaries, } \quad n \geq 2 \text {. }
\end{aligned}
$$

Solving the previous integral equations, the series solutions for the first $N$ terms are given as

$$
u_{N}(x, y, z)=\frac{\sin (z \pi) \sin (\pi y)}{9 \pi^{4}} \sum_{n=0}^{N} \frac{(\pi x)^{2 n+1}}{(2 n+1) !}
$$

Therefore taking the limit at $N$ tending to infinity we obtained

$$
u(x, y, z)=\lim _{N \rightarrow \infty} u_{N}(x, y, z)=\frac{\sin (x \pi) \sin (z \pi) \sin (\pi y)}{9 \pi^{4}} .
$$

\section{Conclusion}

In this paper the recent homotopy decomposition [18-21] is used to solve the $2 \mathrm{D}$ and $3 \mathrm{D}$ Poisson equations and biharmonic equations. The method is chosen because it does not require the linearization or assumptions of weak nonlinearity, 
the solutions are generated in the form of general solution, and it is more realistic compared to the method of simplifying the physical problems. The method does not require any corrected function any Lagrange multiplier and it avoids repeated terms in the series solutions compared to the existing decomposition method including the variational iteration method and the Adomian decomposition method. The approximated solutions obtained converge to the exact solution as $N$ tends to infinity. The numerical values are presented in Table 1 shows that the method is very efficient and accurate.

\section{Acknowledgment}

The authors would like to thank referee(s) for very useful comments regarding the details and their remarks that improved the presentation and the contents of the paper.

\section{References}

[1] R. C. Mittal and S. Gahlaut, "High-order finite-difference schemes to solve Poisson's equation in polar coordinates," IMA Journal of Numerical Analysis, vol. 11, no. 2, pp. 261-270, 1991.

[2] E. Perrey-Debain and H. G. ter Morsche, "B-spline approximation and fast wavelet transform for an efficient evaluation of particular solutions for Poisson's equation," Engineering Analysis with Boundary Elements, vol. 26, no. 1, pp. 1-13, 2002.

[3] G. Sutmann and B. Steffen, "High-order compact solvers for the three-dimensional Poisson equation," Journal of Computational and Applied Mathematics, vol. 187, no. 2, pp. 142-170, 2006.

[4] Y. Ge, "Multigrid method and fourth-order compact difference discretization scheme with unequal meshsizes for 3D Poisson equation," Journal of Computational Physics, vol. 229, no. 18, pp. 6381-6391, 2010.

[5] N. A. Gumerov and R. Duraiswami, "Fast multipole method for the biharmonic equation in three dimensions," Journal of Computational Physics, vol. 215, no. 1, pp. 363-383, 2006.

[6] D. Khattar, S. Singh, and R. K. Mohanty, "A new coupled approach high accuracy numerical method for the solution of 3D non-linear biharmonic equations," Applied Mathematics and Computation, vol. 215, no. 8, pp. 3036-3044, 2009.

[7] I. Altas, J. Erhel, and M. M. Gupta, "High accuracy solution of three-dimensional biharmonic equations," Numerical Algorithms, vol. 29, no. 1-3, pp. 1-19, 2002.

[8] Y. Jeon, "New indirect scalar boundary integral equation formulas for the biharmonic equation," Journal of Computational and Applied Mathematics, vol. 135, no. 2, pp. 313-324, 2001.

[9] N. Mai-Duy and R. I. Tanner, "A spectral collocation method based on integrated Chebyshev polynomials for two-dimensional biharmonic boundary-value problems," Journal of Computational and Applied Mathematics, vol. 201, no. 1, pp. 30-47, 2007.

[10] N. Mai-Duy and T. Tran-Cong, "Solving biharmonic problems with scattered-point discretization using indirect radial-basisfunction networks," Engineering Analysis with Boundary Elements, vol. 30, no. 2, pp. 77-87, 2006.

[11] N. Mai-Duy and R. I. Tanner, "An effective high order interpolation scheme in BIEM for biharmonic boundary value problems," Engineering Analysis with Boundary Elements, vol. 29, no. 3, pp. 210-223, 2005.
[12] X. Li and J. Zhu, "A Galerkin boundary node method for biharmonic problems," Engineering Analysis with Boundary Elements, vol. 33, no. 6, pp. 858-865, 2009.

[13] N. Mai-Duy, H. See, and T. Tran-Cong, "A spectral collocation technique based on integrated Chebyshev polynomials for biharmonic problems in irregular domains," Applied Mathematical Modelling, vol. 33, no. 1, pp. 284-299, 2009.

[14] M. W. M. G. Dissanayake and N. Phan-Thien, "Neural-networkbased approximations for solving partial differential equations," communications in Numerical Methods in Engineering, vol. 10, no. 3, pp. 195-201, 1994.

[15] N. Mai-Duy and R. I. Tanner, "A collocation method based on one-dimensional RBF interpolation scheme for solving PDEs," International Journal of Numerical Methods for Heat \& Fluid Flow, vol. 17, no. 2, pp. 165-186, 2007.

[16] Z. Shi, Y.-Y. Cao, and Q.-J. Chen, "Solving 2D and 3D Poisson equations and biharmonic equations by the Haar wavelet method," Applied Mathematical Modelling, vol. 36, no. 11, pp. 51435161, 2012.

[17] A. Atangana and J. F. Botha, "Analytical solution of the ground water flow equation obtained via homotopy decomposition method," Journal of Earth Science \& Climatic Change, vol. 3, p. 115, 2012.

[18] A. Atangana and A. Secer, "The time-fractional coupled-Korteweg-de-Vries equations," Abstract and Applied Analysis, vol. 2013, Article ID 947986, 8 pages, 2013.

[19] A. Atangana and A. Ahmed, "A generalized version of a low velocity impact between a rigid sphere and a transversely isotropic strain-hardening plate supported by a rigid substrate using the concept of non-integer derivatives," Abstract Applied Analysisaccepted, vol. 2013, Article ID 671321, 9 pages, 2013.

[20] A. Atangana and E. Alabaraoye, "Solving system of fractional partial differential equations arisen in the model of HIV infection of $\mathrm{CD}^{+}$cells and attractor one-dimensional KellerSegel equations," Advance in Difference Equation, vol. 2013, no. 94, 2013.

[21] S. T. Mohyud-Din, A. Yildirim, and M. M. Hosseini, "Variational iteration method for initial and boundary value problems using He's polynomials," International Journal of Differential Equations, vol. 2010, Article ID 426213, 28 pages, 2010.

[22] A. A. Elbeleze, A. Kılıçman, and B. M. Taib, "Homotopy perturbation method for fractional Black-Scholes European option pricing equations using Sumudu transform," Mathematical Problems in Engineering, vol. 2013, Article ID 524852, 7 pages, 2013.

[23] A. A. Elbeleze, A. Kılıçman, and B. M. Taib, "Application of homotopy perturbation and variational iteration method for Fredholm integro-differential equation of fractional order," Abstract and Applied Analysis, vol. 2012, Article ID 763139, 14 pages, 2012.

[24] A. M. A. El-Sayed and D. Hammad, "A reliable treatment of homotopy perturbation method for solving the nonlinear Klein-Gordon equation of arbitrary (fractional) orders)," Journal of Applied Mathematics, vol. 2012, Article ID 581481, 13 pages, 2012.

[25] M. T. Kajani, M. Ghasemi, and E. Babolian, "Comparison between the homotopy perturbation method and the sinecosine wavelet method for solving linear integro-differential equations," Computers \& Mathematics with Applications, vol. 54, no. 7-8, pp. 1162-1168, 2007. 
[26] A. K. Khalifa, "The decomposition method for one dimensional biharmonic equations," International Journal of Simulation and Process Modelling, vol. 2, no. 1-2, 2006. 


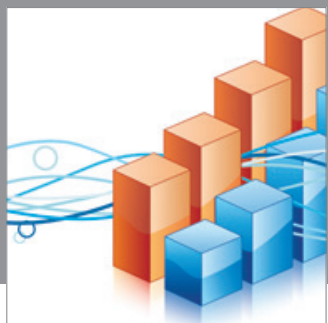

Advances in

Operations Research

mansans

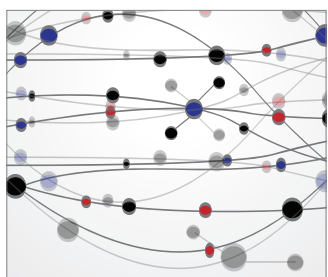

The Scientific World Journal
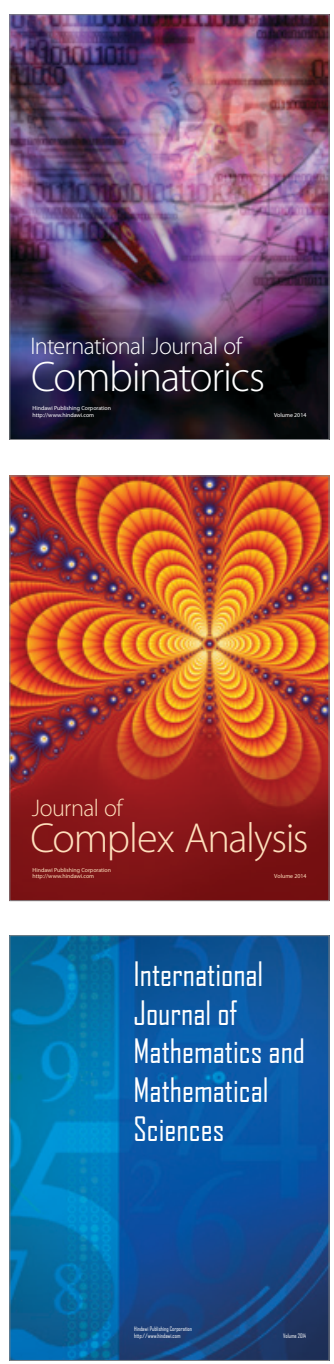
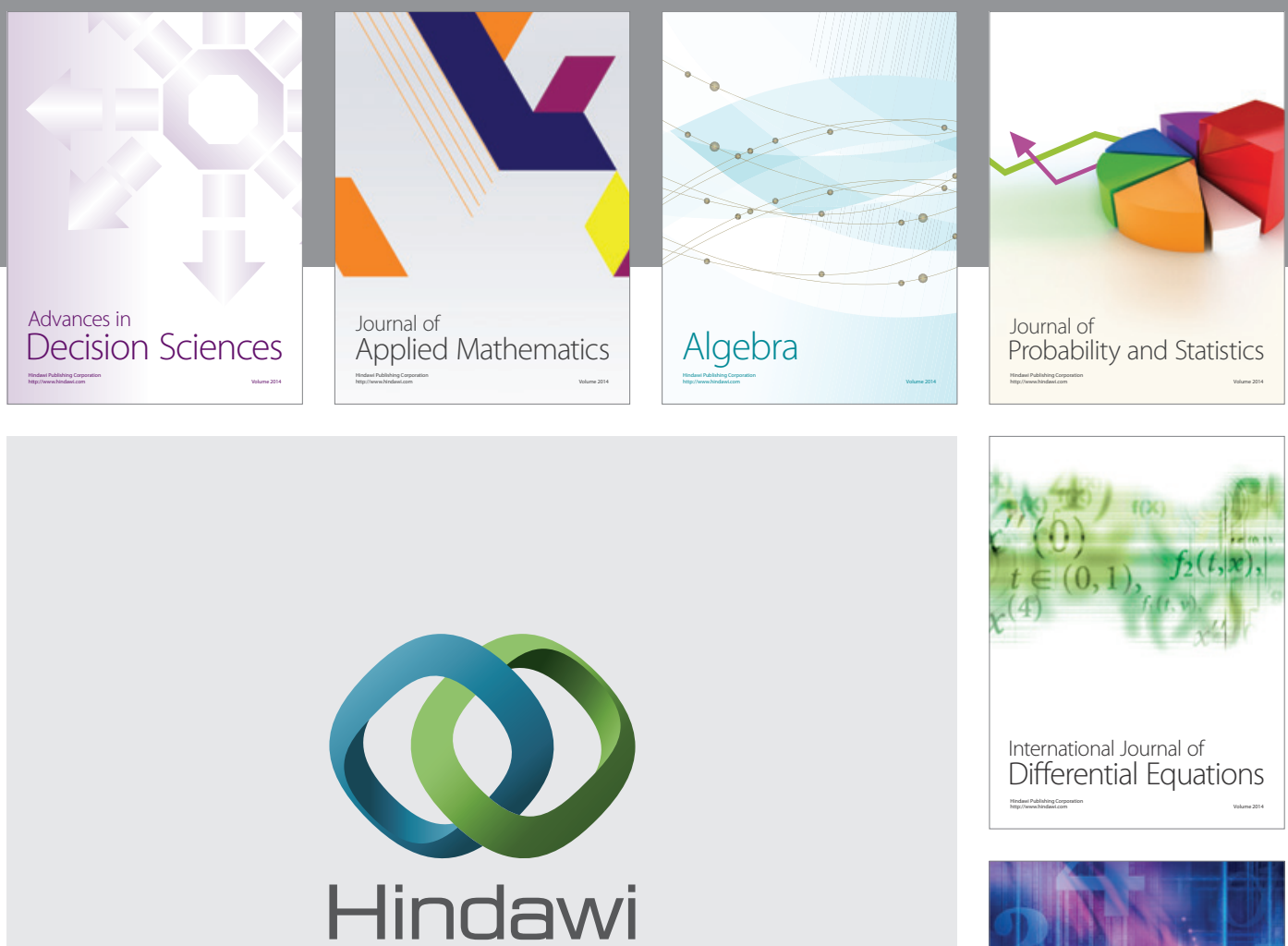

Submit your manuscripts at http://www.hindawi.com
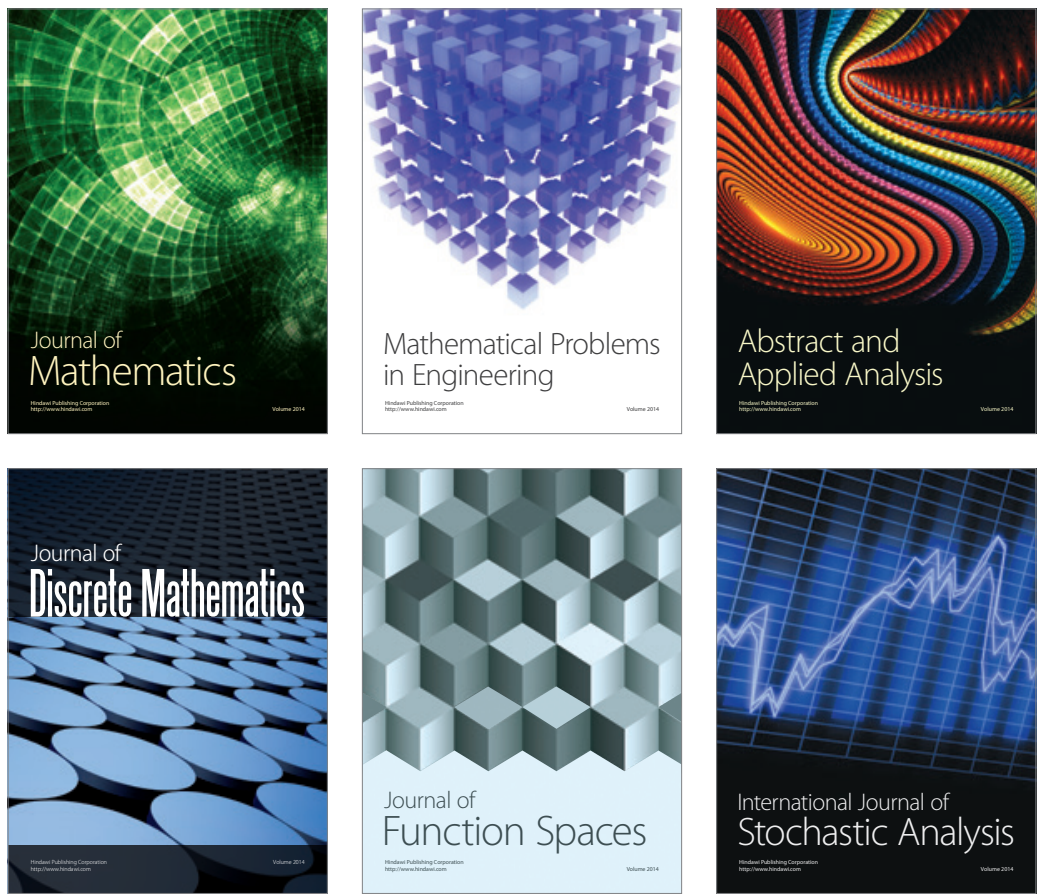

Journal of

Function Spaces

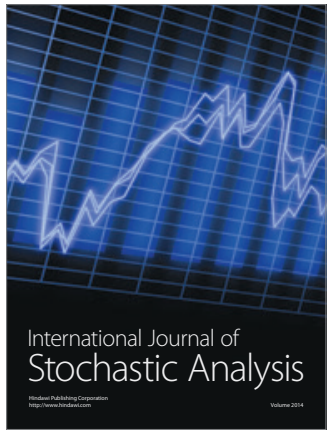

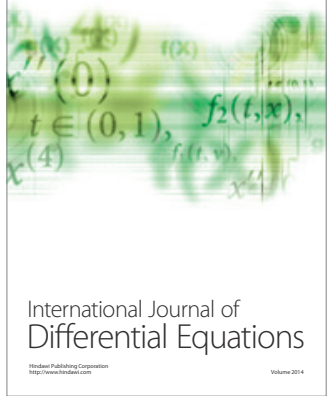
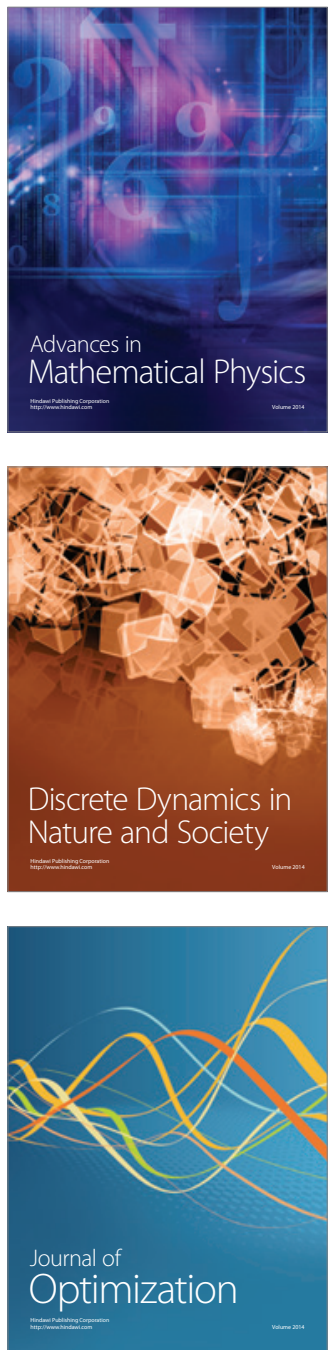